\title{
Laboratory investigations of space-relevant, self-organizing MHD plasmas
}

\author{
P. M. Bellan \\ Caltech, Pasadena CA, 91125
}

\begin{abstract}
Pulsed power technology is used to produce transient MHD-regime plasmas having topology and dynamical behavior similar to solar and astrophysical plasmas. These plasmas are not exact scale models, but exhibit many behaviors similar to actual solar and astrophysical plasmas, for example, collimation, kinking, and jet motion. These plasmas can also display an interaction between MHD-dynamics and non-MHD particle orbit behavior.
\end{abstract}

Keywords: Coronal loops, streamers; Jets, outflows, and bipolar flows; Magnetohydrodynamics and plasmas; Spheromaks

PACS: 96.60.pf, 98.38.Fs, 95.30.Qd, 52.55.Ip

\section{METHODOLOGY}

Because the magnetohydrodynamic (MHD) equations have no intrinsic scale they can be relevant to situations having enormously different scales. For example the MHD equations are relevant to astrophysical situations (scale of $10^{12}-\gg 10^{18}$ meters), the solar corona (scale $10^{6}-10^{9}$ meters), interplanetary space (scale $10^{10}-10^{13}$ meters), planetary magnetospheres (scale $10^{8}$ meters), tokamaks/spheromaks/reverse-field-pinches (scale 0.1 -5 meters), and dense plasma focus devices (scale 0.01-0.1 meters). This vast range of scales suggests that phenomena occurring at one scale can occur at other scales also if the boundary conditions and dimensionless numbers are similar.

Solar and astrophysical MHD-governed plasmas are being simulated in an ongoing experimental research program at Caltech. These experiments replicate one of the most important properties of ideal MHD, namely the freezing of magnetic flux into the frame of a moving plasma. Freezing means that the time for magnetic flux to leak out of the plasma frame is much longer than the time scale of the phenomenon of interest. The frozen-in condition is satisfied by arranging for the experiment duration to be much shorter than the resistive diffusion time. On the other hand, the experimental duration is much longer than the Alfvén time so the experiment lasts long enough for interesting MHD dynamics to occur. The $\sim 10^{3}$ Lundquist number, i.e. ratio of resistive time to Alfvén time, allows for the existence of an experimental time scale short compared to the resistive time, but long compared to the Alfvén time. By imposing various boundary conditions on normal magnetic field, normal mass flux, and electrostatic potential, the topology of specific solar

CP932, Turbulence and Nonlinear Processes in Astrophysical Plasmas

$6^{\text {th }}$ Annual International Astrophysical Conference, edited by D. Shaikh and G. P. Zank

(C) 2007 American Institute of Physics 978-0-7354-0443-4/07/\$23.00 


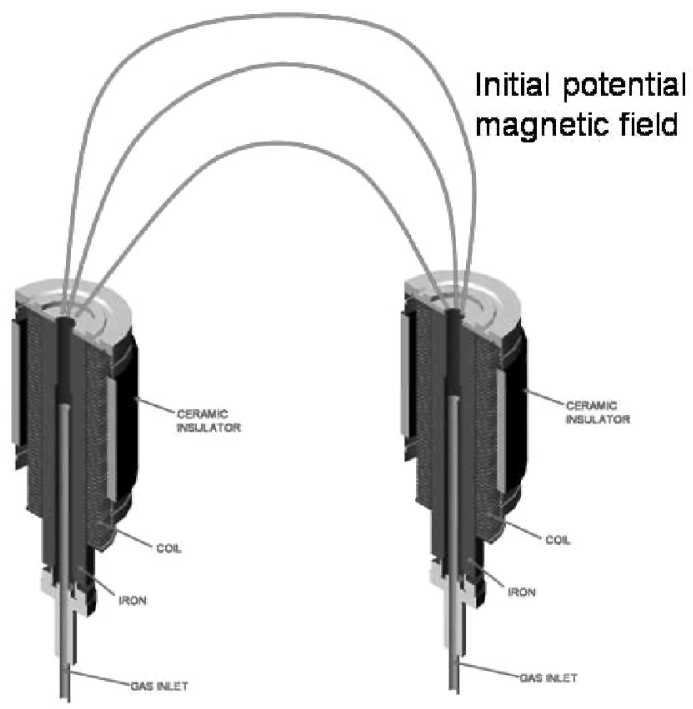

FIGURE 1. Setup for simulation of solar corona loops.

or astrophysical situations can be simulated.

\section{CORONAL LOOP SIMULATIONS}

\section{Configuration and sequence}

Solar coronal loops are simulated using the configuration shown in Fig.1 [1]. The configuration consists of two identical plasma guns, each having a coil behind an orificecontaining electrode. A ceramic cylinder insulates the electrodes from the rest of the system so that an electrostatic voltage drop can be established between the two electrodes. A pulsed gas valve injects gas from the orifice into the vacuum chamber.

The sequence of operation is as follows:

1. The coils are energized ( $10 \mathrm{~ms}$ time scale) with opposite polarity so that a potential magnetic field spans from one electrode to the other.

2. Neutral gas is injected through the orifices on a time scale of approximately $1 \mathrm{~ms}$. 
3. High voltage from a capacitor bank charged to several kilovolts is applied across the electrodes.

4. The low-density neutral gas injected from the orifices breaks down to form a lowdensity plasma in the region between the electrodes.

5. Discharge of the capacitor bank ramps up a large current $(\sim 40 \mathrm{kA})$ that flows through the plasma from one electrode to the other.

6. This current has an associated azimuthal magnetic field linking the original potential magnetic field so the net field becomes twisted.

7. MHD forces accelerate plasma from the orifices into the arched flux tube linking the orifices.

8. The flux tube expands in major radius, contracts in minor radius, and kinks as shown in Fig. 2.

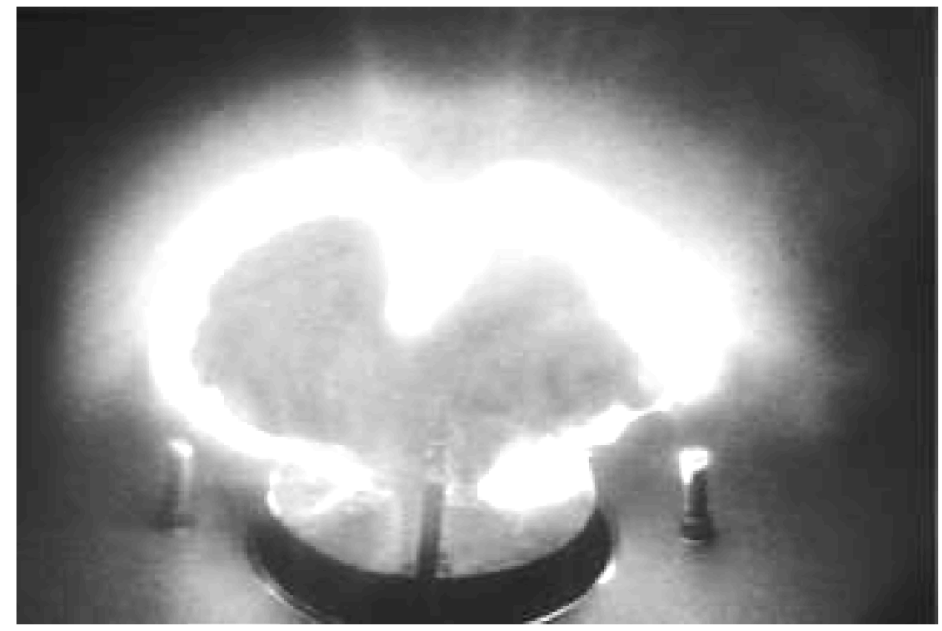

FIGURE 2. Photo of typical plasma produced in solar coronal loop simulation experiment.

\section{Main results}

\section{Strapping field}

An external magnetic field which 'straps down' the erupting loop has been applied [1]. As sketched in Fig.3, this external field $\mathbf{B}_{\text {ext }}$ is at right angles to the loop current $\mathbf{J}_{\text {loop }}$, has a 
TABLE 1. Typical Experimental Parameters

\begin{tabular}{ll}
\hline Parameter & Value \\
\hline experiment duration & $10 \mu \mathrm{s}$ \\
current & $30-60 \mathrm{kA}$ \\
voltage & $3-6 \mathrm{kV}$ at breakdown, $\sim 1 \mathrm{kV}$ after \\
gas & hydrogen, argon, neon, nitrogen \\
density & $\sim 10^{14} \mathrm{~cm}^{-3}$ \\
electron temperature & $2-10 \mathrm{eV}$ \\
camera shutter speed & $10 \mathrm{~ns}$ \\
\hline
\end{tabular}

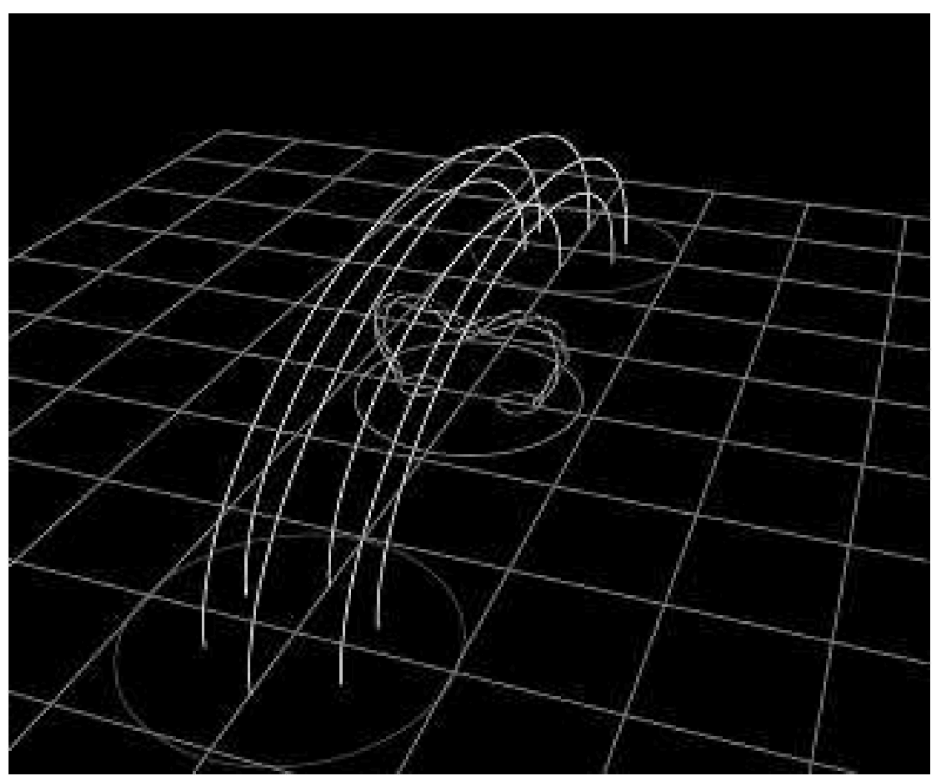

FIGURE 3. Strapping field inhibits expansion of simulated coronal loop.

larger spatial scale than the loop and permeates the loop. The $\mathbf{J}_{\text {loop }} \times \mathbf{B}_{\text {ext }}$ interaction force opposes the loop expansion for appropriate choice of sign of $\mathbf{B}_{\text {ext }}$. If $\mathbf{B}_{\text {ext }}$ is sufficiently strong, loop eruption is reduced or inhibited. 


\section{Laboratory simulation boundary conditions}

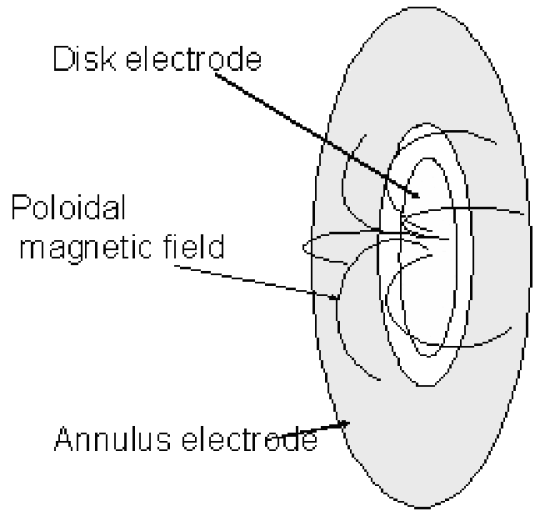

FIGURE 4. Poloidal field links a copper disk to a co-planar copper annulus. Orifices (not shown) in disk and annulus provide sources of plasma.

\section{Co- and counter-helicity merging}

Two pairs of plasma guns have been used to make two adjacent simulated coronal loops having the same or opposite helicity [2]. The current flowing from footpoint to footpoint has the same sense for both loops and so these parallel currents attract. However, the respective directions of the magnetic field lines going from footpoint to footpoint in the two loops can be arranged to be parallel (i.e., co-helicity) or anti-parallel (i.e., counterhelicity). Merging of counter-helicity loops produces a soft x-ray burst about two orders of magnitude larger than the x-rays produced when co-helicity loops merge.

\section{ASTROPHYSICAL JET SIMULATIONS}

Plasmas having the topology of astrophysical jets are produced in an experimental configuration [3] having similar parameters but different geometry compared to the experiment described in Sec.. The astrophysical jet experiment uses a pair of electrodes (anode, cathode) that are co-axial and co-planar as sketched in Fig.4. The cathode is a $20 \mathrm{~cm}$ diameter 
copper disk and the anode is copper annulus with $20.5 \mathrm{~cm}$ inner diameter and $50 \mathrm{~cm}$ outer diameter so there is a slight gap between the co-planar disk and cathode.

A poloidal field is established by a coil located behind the disk-annulus gap. Gas clouds localized in front of the electrodes are established using a set of fast gas puff valves. High voltage applied across the anode-cathode gap breaks down this gas to form an initial lowdensity plasma. MHD forces then ingest additional plasma from the orifices, pump this plasma into the flux tube linking the anode and cathode so that the flux tube becomes dense and bright.

At this stage the plasma consists of eight bright distinct flux tubes each linking an orifice on the cathode to a corresponding orifice on the anode (see Fig.5 top). The major radius of these eight arched flux tubes expands in the first microsecond and then the inner segments of the eight flux tubes merge due to the attraction of the parallel currents (see Fig.5 second from top). The merged segments have the form of a central column. The central column grows in length and becomes very jet-like (see Fig. 5 third from top). The central column becomes kink-unstable [4] at a critical length consistent with the predictions of the Kruskal-Shafranov kink stability theory (see Fig. 5 bottom).

\section{COLLIMATION MODEL}

Both the solar coronal loop and the astrophysical jet plasmas are observed to be highly collimated. This collimation develops quickly and persists through the dynamics. The pervasiveness of collimation suggests some fundamental and ubiquitous dynamics is responsible.

A model has been developed [5] that explains collimation in terms of the pile-up of frozen-in azimuthal magnetic flux. The induction equation shows that flux is frozen into the frame of the plasma (recall that flux is defined by drawing an arbitrary closed contour in the frame of the plasma and then 'counting' the number of magnetic field lines linked by this contour). The azimuthal flux associated with an electric current flowing along a flux tube can be imagined as a set of elastic bands wrapped around the cylindrical plasma. The jet-motion of the plasma in the axial direction and the frozen-in condition implies that these elastic bands can be considered as being frozen into the plasma frame and hence moving axially with the plasma jet.

If the plasma jet velocity has an axial dependence such that the axial flow is converging, then there will be a pile-up of mass at the axial location of convergence (see Fig.6). Because the 'elastic bands' were frozen into the flow, the elastic bands will also pileup. The pile-up of elastic bands is tantamount to an increase in the density of azimuthal magnetic field lines, i.e., an increase in $B_{\phi}$. Since $B_{\phi} r \sim I$ where $I$ is the axial current, and since $I$ is fixed by the current flowing along the flux tube, increasing $B_{\phi}$ causes $r$ to decrease. Thus, there is a reduction of flux tube radius at locations where the flow has an axial convergence. This constitutes a convective extension of the pinch phenomenon. 


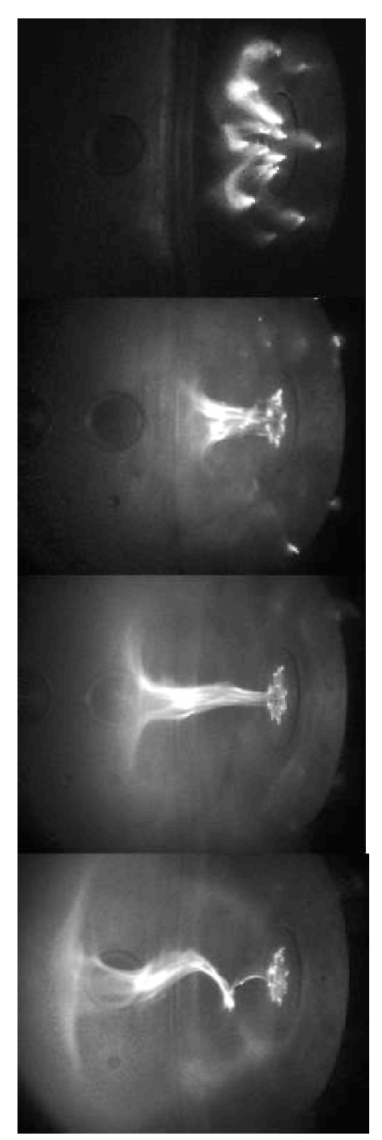

FIGURE 5. Jet formation and evolution sequence.

Because flows are accelerated near the electrodes, but then slow down on moving away, the collimation effect is expected to be very common. Detailed measurements of collimation made in astrophysical jet experiments [6] support the idea that collimation results from the stagnation of MHD flows carrying frozen-in magnetic flux. 


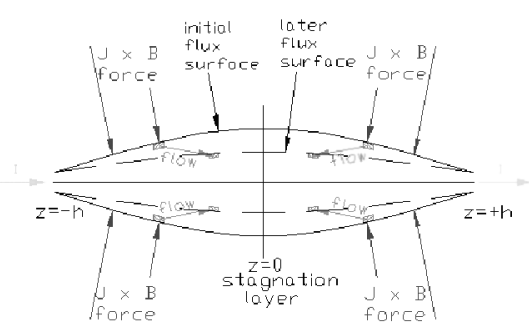

FIGURE 6. MHD jets driven by $\mathbf{J} \times \mathbf{B}$ flows converge and pile-up of frozen-in azimuthal magnetic field lines increases $B_{\phi}$ causing collimation.

\section{TWO-GAS EXPERIMENTS}

Experiments have recently been initiated where different gases are injected at different orifices. This method is being used in both the coronal loop and the astrophysical jet simulation experiments.

In the astrophysical jet experiment, a cloud of neutral gas is placed in the path of the plasma jet and the jet ionizes the target gas cloud upon impact. Distinct behaviors are observed depending on whether the target gas is lighter or heavier than the jet gas. The jet and the target are imaged separately using optical line filters in front of the camera lens. The two images are then superimposed to create a composite, color-coded image so that the jet and the target can be distinguished from each other and the temporal motion of their interface can be examined.

The solar coronal loop simulation experiment is arranged so that different gases are injected at the cathode and anode footpoints. Plasma jets start at the orifices at the footpoints, accelerate from the footpoints, and then collide. For example, it is observed that when hydrogen is injected at one footpoint and nitrogen at the other, a hydrogen jet flows from its footpoint and a nitrogen jet flows from its footpoint. The two jets propagate along the flux tube and then collide with each other. The hydrogen jet goes much faster than the nitrogen jet so the collision occurs nearer the nitrogen footpoint. The flux tube is thus one color on one side of the collision region and another color on the other side. The flux tube is collimated and expands in major radius just like a flux tube produced with a single gas.

\section{KINETIC JETS}

An unexpected phenomenon observed in the coronal loop experiment involves a synergism between MHD and non-MHD physics. Plasma jets are accelerated by MHD forces (i.e., 
the $\mathbf{J} \times \mathbf{B}$ term in the MHD equation of motion). These jets can propagate from a cathode or from an anode since the jet acceleration is essentially due to a force $J_{r} B_{\phi} \sim-\partial B_{\phi}^{2} / \partial z$ which is insensitive to the sign of $B_{\phi}$. Thus the solar loop simulation experiment has jets propagating from both cathode and anode footpoints.

Plasma jets propagating from the cathode seem paradoxical from the single particle point of view, because the ions which comprise most of the plasma mass would have to be accelerated from the cathode. This means that a positive particle is being accelerated from a negative electrode. There is in fact no paradox because electrons travel faster from the cathode than do the ions. The ions must follow the electrons in order to maintain quasineutrality. The result is that, in accordance with its definition, the conventional electric current flows from anode to cathode, even though the plasma (comprised of both electrons and ions) is flowing away from the cathode. Thus, the particle flow is opposite the electric current flow. The ions do not flow from cathode to anode, but instead flow from both cathode and anode to the region between cathode and anode and accumulate in the region between cathode and anode. The electrons flow in whatever way is necessary to maintain quasi-neutrality.

The ions near the cathode are thus flowing opposite to the direction of conventional electric current flow. This results in a radially outwards force acting on individual ions by virtue of their magnetic interaction with the magnetic field produced by the conventional current. In a sense the counter-current ions can be considered an anti-parallel current that is repelled by the conventional current.

This details of this process and the criterion for instability onset can be understood by considering the radial component of the ion equation of motion in a helical magnetic field $\mathbf{B}=B_{\phi} \hat{\phi}+B_{z} \hat{z}$. This radial component is

$$
m \ddot{r}-\frac{m v_{\phi}^{2}}{r}=q\left(v_{\phi} B_{z}-v_{z} B_{\phi}\right)
$$

where the second term on the left is the centrifugal force. If there is no current, then $B_{\phi}=0$ and if $\ddot{r}=0$ is assumed, then the equation reduces to $\dot{\phi}=v_{\phi} / r=-q B_{z} / m$ which is just the classic cyclotron (Larmor) orbit. However, if there is a current so $B_{\phi} \neq 0$, then if $\ddot{r}=0$ is assumed, Eq. 1 becomes

$$
\dot{\phi}^{2}+\dot{\phi} \frac{q B_{z}}{m}-v_{z} \frac{q B_{\phi}}{m r}=0
$$

a quadratic equation in $\dot{\phi}$. Near the $z$ axis, the axial current can be considered approximately radially uniform so

$$
B_{\phi}=\mu_{0} J_{z} r / 2
$$


The two solutions of Eq. 2 are thus

$$
\dot{\phi}=\frac{-\frac{q B_{z}}{m} \pm \sqrt{\left(q B_{z} / m\right)^{2}+2 v_{z} q \mu_{0} J_{z} / m}}{2}
$$

and there is no real solution if the argument of the square root is negative, i.e., if

$$
\frac{1}{2}+\frac{\mu_{0} J_{z}}{B_{z}} \frac{m v_{z}}{q B_{z}}<0
$$

Thus, if $q v_{z}$ has the opposite sign of $J_{z}$ (i.e., the ions flow in the direction opposite to the conventional current direction) and if the ion velocity is sufficiently fast, there is no real solution to the equation of motion for constant $r$. In this case $r$ cannot be constant and examination of Eq. 1 shows that when $q v_{z} B_{\phi}$ is large and negative, $\ddot{r}$ is positive, i.e., the ion is expelled radially from the flux tube.

This behavior has been analyzed in more detail [7] using the Hamilton-Lagrange method. It is found that the instability corresponds to a radial effective potential changing from being a valley to being a hill. Direct numerical solution of the equation of motion verifies the existence of this instability and the conditions for its onset. Detailed experimental measurements [7] show that the instability threshold occurs in a manner consistent with Eq. 5.

\section{ACKNOWLEDGMENTS}

This paper summarizes work done in collaboration with J. F. Hansen, S. C. Hsu, S. K. P.

Tripathi, G. S. Yun, S. You, A. Moser, and E. Stenson. Supported in part by USDOE and NSF.

\section{REFERENCES}

1. J. F. Hansen, and P. M. Bellan, Astrophysical Journal 563, L183-L186 (2001), part 2.

2. J. F. Hansen, S. K. P. Tripathi, and P. M. Bellan, Physics of Plasmas 11, 3177-3185 (2004).

3. S. C. Hsu, and P. M. Bellan, Monthly Notices of the Royal Astronomical Society 334, 257-261 (2002).

4. S. C. Hsu, and P. M. Bellan, Physical Review Letters 90, 215002 (2003).

5. P. M. Bellan, Physics of Plasmas 10, 1999-2008 (2003), part 2.

6. S. You, G. S. Yun, and P. M. Bellan, Physical Review Letters 95, 045002 (2005).

7. S. K. P. Tripathi, P. M. Bellan, and G. S. Yun, Physical Review Letters 98, art. no. 135002 (2007). 